\title{
Assessment of maternal deaths due to chronic hypertension: Lessons to learn - a 'red flag' for maternal and fetal complications
}

\author{
J Moodley, ${ }^{1}$ MB ChB, FCOG (SA), FRCOG, MD; N C Ngene, ${ }^{2}$ MBBS, Dip Obst (SA), Dip HIV Man (SA), MMed (Fam Med), \\ FCOG (SA), MMed (O\&G) \\ ${ }^{1}$ Women's Health and HIV Research Group, Department of Obstetrics and Gynaecology, School of Clinical Medicine, College of Health Sciences, \\ University of KwaZulu-Natal, Durban, South Africa \\ ${ }^{2}$ Department of Obstetrics and Gynaecology, Klerksdorp Hospital, South Africa
}

Corresponding author:J Moodley (jmog@ukzn.ac.za)

Hypertension, including chronic hypertension and gestational hypertension, is the most common medical complication in pregnancy. Chronic hypertension is associated with up to $5 \%$ of all pregnancies and accounts for a significant number of adverse complications, such as superimposed pre-eclampsia, abruptio placentae, iatrogenic preterm labour, stillbirth, small-for-gestational-age fetus and maternal death. To improve care, women with chronic hypertension should seek professional advice before pregnancy. Antihypertensive agents, which are known to cause fetal abnormalities, should be replaced with drugs safe for use in pregnancy. Alternatively, a safe antihypertensive agent should be offered as soon as pregnancy is confirmed. Furthermore, women with chronic hypertension have a 13 - $40 \%$ chance of developing superimposed pre-eclampsia and need to be seen more frequently during the antenatal period, either by a specialist or under their supervision.

S Afr Med J 2018;108(11):896-900. DOI:10.7196/SAMJ.2018.v108i11.13365

Chronic hypertension affects up to $5 \%$ of pregnant women in the USA. ${ }^{[1]}$ More importantly, its prevalence may be increasing owing to increasing obesity, women choosing to become pregnant in the later period of child-bearing age and an increasing number of pregnancies with comorbidities, such as diabetes and renal disease. ${ }^{[2]}$

Chronic hypertension may occur more frequently in black population groups than in the white population. It is a risk factor for adverse maternal and perinatal outcomes, and $13-40 \%$ of women may develop superimposed pre-eclampsia. ${ }^{[1]}$ This article presents data on lessons that may be learnt from maternal death assessments and discusses a practical approach to the clinical management of chronic hypertension in pregnancy.

\section{Data of maternal deaths from chronic hypertension in $2014-2016$ in South Africa}

Chronic hypertension is one of the categories of the hypertensive disorders of pregnancy (HDP), the others being gestational hypertension, white-coat hypertension and pre-eclampsia, which may be de novo or superimposed on chronic hypertension, eclampsia, and haemolysis, elevated liver enzymes and low platelet count (HELLP) syndrome. ${ }^{[3]}$ Recently, the International Society for the Study of Hypertension in Pregnancy suggested that clinicians should be aware of two entities, i.e. masked hypertension and transient gestational hypertension. ${ }^{[3]}$ Masked hypertension is defined as a normal blood pressure (BP) level recorded in a health facility but an elevated BP outside such a facility, while transient gestational hypertension denotes de novo hypertension and spontaneous resolution during pregnancy. ${ }^{[3]}$

There were 661 maternal deaths due to HDP in South Africa (SA) in the triennium $2014-2016 \cdot{ }^{[4]}$ In $40(6 \%)$ of these deaths, assessors ascertained that chronic hypertension was the primary cause of death. The clinical characteristics of these deaths are shown in Table 1.

All patients were $>28$ years of age and $22(55 \%)$ were $>35$ years old. There were 4 primigravidas between the ages of 28 and 34 years.

Table 2 shows preventable factors and indicates that these were mainly related to the conduct of health professionals.

Maternal deaths due to chronic hypertension occurred at all levels of healthcare - public sector (district and regional hospitals) and private sector. Seven patients were readmitted to hospital after delivery; they are particularly prominent owing to the following reasons related to their management: early hospital discharge without good control of high BP, and failure to recognise that respiratory signs in women with chronic hypertension in the postpartum period may signify pulmonary oedema or peripartum cardiomyopathy. In a proportion of cases, there was failure to recognise the likelihood of cardiopulmonary problems, with resultant delay in investigation and diagnosis. Therefore, the main problems in clinical management can be summarised as follows: (i) failure to identify patients at risk of complications; (ii) failure to triage patients or seek specialist advice timeously; (iii) delays in transport for referral to a higher level of healthcare; and (iv) delays in immediate appropriate treatment of high BP.

\section{Clinical management of chronic hypertension in pregnancy}

Chronic hypertension is defined as systolic/diastolic BP $\geq 140 / 90 \mathrm{mmHg}$, documented before pregnancy or before the 20th week of pregnancy on at least two occasions at least 4 hours apart. ${ }^{[1]}$ Many women of childbearing age only commence antenatal visits or antenatal care during the second or third trimester of pregnancy, particularly in low- and middle-income countries. Given the physiological changes in the first 
Table 1. Clinical characteristics of 40 patients who died owing to chronic hypertension, 2014 - 2016

\begin{tabular}{ll}
\hline Clinical characteristics & $\boldsymbol{n}(\%)$ \\
\hline Age (mean 33, range 28 - 45), years & 9 \\
$28-30$ & 9 \\
$31-34$ & 14 \\
$35-39$ & 8 \\
$\geq 40$ & 4 \\
Parity (mean 3, range 0 - 6) & 20 \\
0 & 2 \\
$1-3$ & \\
$\geq 4$ & 8 \\
Twin pregnancy (gestational age on admission) (mean 33, range 7 - 36), weeks & 3 \\
$<20$ & 27 \\
$<20$ (with severe hypertension, requiring termination of pregnancy) & 5 \\
$21-33$ & 7 \\
$<34$ & 3 \\
Hospital readmission & 2 \\
Severe hypertension & 2 \\
Pulmonary oedema & \\
Respiratory distress & \\
Antenatal care & \\
Booked for antenatal care & $30(75)$ \\
HIV-positive & $18(45)$ \\
HIV unknown & $5(13)$ \\
Vaginal delivery & $20(50)$ \\
Caesarean delivery & $20(50)$ \\
History of chronic hypertension & $5(13)$ \\
& $5(13)$
\end{tabular}

half of pregnancy, i.e. a decrease in BP owing to vasodilation, ${ }^{[5]}$ high $\mathrm{BP}$ recorded for the first time in the late second or third trimester is usually diagnosed as gestational hypertension. This could be chronic hypertension, but it can only be confirmed after the puerperium, although it may take up to 12 weeks post partum. ${ }^{[6]}$ To avoid this, all women planning a pregnancy should attend a preconception clinic or visit a healthcare professional to exclude diseases such as hypertension or diabetes and optimise their disease condition before becoming pregnant. ${ }^{[6]}$

High BP levels in pregnancy are usually regarded as mild (systolic $140-159 \mathrm{mmHg}$, and/or diastolic $90-109 \mathrm{mmHg}$ ), or severe (systolic $\geq 160 \mathrm{mmHg}$ and/or diastolic $\geq 110 \mathrm{mmHg}$ ). ${ }^{[1]}$ Women with severe hypertension are at risk of developing superimposed pre-eclampsia, which may occur in up to $30 \%$ of cases in the second half of pregnancy. ${ }^{[7]}$

Most patients with chronic hypertension have essential or primary hypertension and are treated as having this condition. However, the following patients require investigation to exclude secondary causes, such as renal disease, collagen vascular and endocrine disorders: young women, patients whose high BP levels are difficult to control and/or require $\geq 3$ antihypertensive agents, and those whose BP levels vary widely. Similarly, women who have been labelled as having gestational hypertension, particularly severe hypertension, and whose BP levels do not settle 12 weeks post partum, require further investigation.

Moreover, in women presenting with chronic hypertension in pregnancy, investigations should be done to exclude comorbidities, including pregestational diabetes. Box 1 shows the investigations required.

In general, initial assessments may help to differentiate between low- and high-risk cases of chronic hypertension. The latter would
Table 2. Probable preventable factors in maternal deaths due to chronic hypertension

Patient-related factors

Declined admission and/or advice $(n=5 ; 12.5 \%)$

Health professions (medical doctors and professional nurses)related factors*

Poor history taking

Poor/incomplete physical examination of patient

Delay in interventions

Delay in obtaining specialist advice

Timeous referrals

Postnatal care/advice

${ }^{*}$ All of these preventable factors occurred in combinations.

\section{Box 1. Assessment and investigation}

A detailed history and physical examination, including current antihypertensive agents, previous surgery and previous pregnancy outcomes

A screening glucose tolerance test and/or haemoglobin Alc to exclude pregestational diabetes

An echocardiograph to exclude cardiac structural defects, such as left ventricular hypertrophy

An electrocardiogram (ECG), particularly in those who have had chronic hypertension for $>5$ years, who previously had superimposed pre-eclampsia, and/or who were readmitted for severe hypertension and pulmonary oedema during a previous pregnancy Proteinuria, either by a spot urine protein:creatinine ratio or a 24-hour urine proteinuria quantitative test. These are important to obtain for a baseline record 
include women with severe hypertension (systolic or diastolic), previous pregnancy losses and/or superimposed pre-eclampsia. High-risk chronic hypertensive patients should be seen more frequently during the antenatal period and advised that they might require hospitalisation if they develop superimposed pre-eclampsia. Warning signs may be the presence of proteinuria and slight rises in BP, especially $5 \mathrm{mmHg}$ increases in diastolic pressure before 20 weeks'

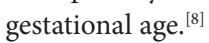

Although some authors divide chronic hypertension in pregnancy into low and high risk, ${ }^{[6,7]}$ even low-risk patients have a $10 \%$ chance of developing superimposed pre-eclampsia and an increased chance of abruptio placentae, premature delivery and perinatal death compared with normotensive pregnant women; therefore, it may be appropriate in the SA setting to obtain specialist advice for all patients, regardless of low- or high-risk, and especially when there are cases of comorbid disease, such as renal and collagen vascular disease. ${ }^{[9]}$ Prophylactic aspirin and supplemental calcium therapies, if started timeously (at end of the first trimester), may assist in preventing superimposed pre-eclampsia.

There is general consensus in the adult population that long-term hypertension is associated with risks, particularly stroke, and that long-term BP control decreases the risks of myocardial infarction, stroke and renal disease. ${ }^{[10]}$

More recently, there has been a call to initiate antihypertensive agents at BP levels of $130 / 80 \mathrm{mmHg}$ if the 10-year risk of a cardiovascular event exceeds $10 \% .{ }^{[11]}$

There is consensus that severe hypertension in pregnancy should be treated because of the risk of haemorrhagic stroke. ${ }^{[1]}$ However, there is controversy with regard to treatment of mild-to-moderate elevations in BP because of exposure of the fetus to antihypertensive agents, even if it is over a relatively short period and without any evidence of benefit to the mother. ${ }^{[1]}$ This controversy continues, ${ }^{[12]}$ despite the Control of Hypertension in Pregnancy Study (CHIPS) - a large randomised trial that compared tight control $(<85 \mathrm{mmHg}$ diastolic BP) with less-tight control $(<100 \mathrm{mmHg}$ systolic BP) in women with both chronic and gestational hypertension; the primary outcomes of the study showed no differences, which included superimposed pre-eclampsia, fetal growth restriction, preterm birth and abruptio placentae, although the rate of progression to severe hypertension was significantly higher in the less-tight group.

Women with chronic hypertension during pregnancy may present in a number of ways:

- If the woman presents in pregnancy and is receiving antihypertensive agents, it is best to discontinue these drugs, which are contraindicated during pregnancy. Examples include angiotensin-converting enzyme inhibitors and angiotensinreceptor blockers, which are associated with renal and craniofacial abnormalities of the fetus. ${ }^{[1]}$ If the BP levels are mildly elevated, take a wait-and-see approach, and restart antihypertensives once the BP becomes elevated. Methyldopa is probably the drug of choice, because it is reported to have no harmful fetal effects, but it may not be as effective as labetalol and calcium channel blockers (Table 3).

- Women presenting with severe hypertension in pregnancy ( $\geq 160 \mathrm{mmHg}$ systolic BP or $>110 \mathrm{mmHg}$ diastolic BP), should receive oral antihypertensive treatment (including a rapidacting agent) immediately.

- Women presenting with chronic hypertension and/or superimposed pre-eclampsia, require additional care that usually involves admission to hospital, stabilisation of high BP with antihypertensive agents (Table 3), including rapid-acting agents, complete laboratory investigations and a management plan, including timing of delivery.

\section{Timing of delivery}

This is usually individualised; inpatients with well-controlled hypertension are mostly delivered at 39 weeks' gestational age. ${ }^{[3]}$ Those with superimposed pre-eclampsia are usually delivered by the end of the 36th week of pregnancy or earlier, depending on the individual case. The decision for delivery should be taken by an obstetrician experienced in the management of HDP.

\section{Postpartum management of chronic hypertension}

Women with high-risk hypertension (severe chronic hypertension, chronic hypertension with superimposed pre-eclampsia, chronic hypertension with multiple antihypertensive agents, chronic hypertension that developed to abruptio placentae or comorbid disease) should be monitored closely for development of serious complications, such as pulmonary oedema, congestive heart failure and renal failure.

It should be remembered that because of fluid shifts from the extra- to intravascular compartments immediately after pregnancy, there are BP elevations in both diastolic and systolic components in normotensive and hypertensive women. These incremental rises in BP occur mainly in the first 4 days post partum. It is therefore not surprising that the International Society for the Study of Hypertension in Pregnancy recommends that BP should be monitored closely in all high-risk patients with hypertensive disorders for at least 3 days after delivery ${ }^{[3]}$ This is of importance because, in the SA setting, patients

Table 3. Antihypertensive drugs commonly used in South Africa

\begin{tabular}{|c|c|c|c|}
\hline Antihypertensive drug & Mechanism of action & Dose & Side-effects \\
\hline \multicolumn{4}{|l|}{ Maintenance agent } \\
\hline Methyldopa & $\alpha_{2}$-receptor & $500-3000 \mathrm{mg} 2-3 \times$ daily & Elevation in liver enzymes, depression \\
\hline Nifedipine, extended release & $\begin{array}{l}\text { Dihydrapone calcium channel } \\
\text { blocker }\end{array}$ & $30-120$ mg orally daily & $\begin{array}{l}\text { Headache, flushing, reflex tachycardia, } \\
\text { peripheral oedema, increased liver } \\
\text { enzymes }\end{array}$ \\
\hline Amlodipine & Calcium channel blocker & $2.5-10.0 \mathrm{mg}$ daily & As above \\
\hline \multicolumn{4}{|l|}{ Rapid-acting antihypertensive agents } \\
\hline Labetalol & Non-selective $\beta$-blocker & $\begin{array}{l}20 \mathrm{mg} \text { intravenously, } \\
\text { then } 40 \mathrm{mg} \text {, then } 80 \mathrm{mg} \\
\text { every } 10 \mathrm{~min} \\
\text { Maximum dose } 300 \mathrm{mg}\end{array}$ & $\begin{array}{l}\text { Bronchospasm } \\
\text { Do not use in congestive cardiac } \\
\text { failure }\end{array}$ \\
\hline Nifedipine, immediate release & Calcium channel blocker & $\begin{array}{l}10 \mathrm{mg} \text { orally every } 20 \mathrm{~min} \\
\text { for } 3 \text { doses }\end{array}$ & Headache, flushing, reflex tachycardia \\
\hline
\end{tabular}


with HDP are discharged as soon as possible and are seen after delivery at lower levels of healthcare (community clinics), which has resulted in a number of cases of maternal deaths due to pulmonary oedema and cardiomyopathy in the postpartum period. ${ }^{[4]}$

Women with severe chronic hypertension and those with superimposed pre-eclampsia must be monitored closely (BP, pulse, respiratory rate) and chest radiographs and electrocardiograms (ECGs) should be done for at least 72 hours, if not done in the antenatal period.

There may be advantages in using angiotensin-converting enzyme inhibitors in the postpartum period, especially if there is a comorbid condition such as diabetes or cardiomyopathy. In such cases, angiotensin-converting enzyme inhibitors, such as enalapril and captopril, have been studied most in pregnancy and have shown to be safe during breastfeeding. ${ }^{[12]}$

Other antihypertensive agents used during breastfeeding and found to be safe are diuretics and beta-blockers, such as labetalol and nifedipine. The latter agent is thought to be excellent during the postpartum period, given its reno-protective effects, and is the preferred first-line agent in the postpartum period. Despite the lack of robust studies to direct the choice of antihypertensive agents in the postpartum period, a physician's familiarity with antihypertensive agents and the past drug history of the patient should be given consideration during drug prescription. There is concern that the use of diuretics may reduce intravascular volume and decrease lactation; however, their use must be based on the compelling need to use diuretics in pulmonary oedema and uncontrolled hypertension in pregnancy. The authors, however, prefer a calcium channel blocker as a first-line antihypertensive agent.

Contraceptive advice is of particular importance, given that most women with essential chronic hypertension are in the latter part of reproductive life. In the SA setting, postpartum insertion of an intrauterine contraceptive device prior to discharge should be considered, or male and female contraceptive methods discussed during the antenatal period, including tubal ligation, as the caesarean delivery rate in high-risk chronic hypertension is $>50 \% .{ }^{[4}$

\section{Summary}

- Chronic hypertension has a significant maternal and neonatal mortality and morbidity.

- Advice on the management of chronic hypertension in pregnancy must be obtained from a specialist or health professional experienced in the management of HDP.

- Most patients with chronic hypertension who are regarded as being at risk, require antihypertensive agents and repeated hospitalisations, and are delivered before 37 weeks of pregnancy.

- Postpartum management of chronic hypertension needs to take into account that there are small but important incremental rises in systolic and diastolic BP, mainly in the first 3 days post partum.

- Consideration must be given to an appropriate antihypertensive agent during breastfeeding.

\section{Case vignette}

A 32-year-old patient (parity 3, gravida 4) with a history of chronic hypertension was referred by a general medical practitioner to a regional hospital. She had initially booked at a community clinic but was not referred, despite a history of chronic hypertension and a previous caesarean section. She had severe hypertension (BP 160/115 $\mathrm{mmHg}$ ), proteinuria (4+), and was obese. Because her BP was difficult to control, she was referred to a tertiary hospital a day later. Prior to referral, magnesium sulphate was administered. The patient went into spontaneous labour at the tertiary hospital and delivered a stillborn baby (1 $400 \mathrm{~g})$. The patient was referred back to the regional hospital after 14 days, but her BP was not well controlled. She was admitted for 5 days for $\mathrm{BP}$ control and then discharged, having to return in a month. She returned 8 days later with a history of dyspnoea, sweating and difficulty with breathing. She was managed in a postnatal ward on a combination of antihypertensive agents and died 5 days later.

\section{Comments}

There was suboptimal care in the management of this patient:

- At the time of antenatal care booking at the community clinic, she gave a history of chronic hypertension and previous caesarean section; she was also obese. She should have been referred to a regional hospital for a full assessment of her cardiovascular system and a screening glucose tolerance test. All patients with chronic hypertension in pregnancy must be referred to a higher level of care or high-risk clinic for assessment, advice and plan of management, including the level of healthcare for further antenatal care.

- On referral to the regional hospital, she had severe proteinuric hypertension at 32 weeks' gestation. There was a delay in her being seen by a medical doctor. She was administered magnesium sulphate and transferred to a tertiary hospital. There were scanty notes regarding the exact diagnosis and communication with medical staff at the tertiary hospital. This patient probably had severe superimposed pre-eclampsia or chronic hypertension, and should have been delivered.

- At the tertiary hospital, it appears that although she had severe preeclampsia with a BP that was difficult to control, she was treated conservatively. Despite this, she was referred back to the regional hospital.

- The tertiary hospital should have investigated her fully to exclude a secondary cause, given the difficulty in controlling her high BP.

- There was no evidence of any investigations for excluding renal/ endocrine/immunological causes of her chronic hypertension or of a combined medical physician/obstetrician being consulted.

- There is a need to strengthen inter-facility communication when there is referral upstream and downstream.

- There was failure of doctors to recognise worsening of HDP (chronic hypertension).

This case illustrates suboptimal care highlighted by the following points:

- Patients with chronic hypertension must be referred to a specialist or experienced medical officer for advice.

- There is a need to strengthen inter-facility communication when patients are referred.

- Uncontrollable hypertension, especially following delivery, requires a multidisciplinary team approach to management.

- Superimposed pre-eclampsia or chronic hypertension is a 'red flag, and a more aggressive approach to timing of delivery must be taken. In this case, there was severe hypertension and proteinuria at 32 weeks' gestation. Consideration should have been given to delivery.

\section{Declaration. None.}

Acknowledgements. The South African National Department of Health for giving permission to publish the information drawn from an assessment of maternal deaths due to chronic hypertension. JM is the Chair of the Committee on Confidential Enquiries into Maternal Deaths. We appreciate the assistance of Dr Margaret Olutayo Alese with the editing of this article. 
Author contributions. Both authors contributed to the writing and proofreading of the article. JM analysed the data and did the assessment of all cases related to death.

Funding. None.

Conflicts of interest. None.

1. American College of Obstetricians and Gynecologists. Hypertension in Pregnancy. Report of the American College of Obstetricians and Gynecologists' Task Force on Hypertension in Pregnancy. Obstet Gynecol 2013;122(5):1122-1131. https://doi.org/10.1097/01.AOG.0000437382.03963.8851-61

2. Sealy EW, Ecker J. Chronic hypertension in pregnancy. N Engl J Med 2001;265:439-446. https://doi. org/10.1056/NEJMcp 0804872

3. Brown MA, Magee LA, Kenny LC, et al. The hypertensive disorders of pregnancy: ISSHP classification, diagnosis and management recommendations for international practice 2018. Pregnancy Hypertens 2018;13:291-310. https://doi.org/10.1016/j.preghy.2018.05.004

4. National Department of Health. Saving Mothers Report: 2014 - 2016. Pretoria: NDoH, 2015

5. Ngene NC, Moodley J. Physiology of blood pressure relevant to managing hypertension in pregnancy. J Matern Fet Neon Med 2017;27:1-10. https://doi.org/10.1080/14767058.2017.140456

6. Chahine KM, Sibai BM. Chronic hypertension in pregnancy: New concepts for classification an management. Am J Perinatol 2018; (epub ahead of print). https://doi.org/10.1055/s-0038-1666976
7. Ankumah NE, Sibai BM. Chronic hypertension in pregnancy: Diagnosis, management and outcomes. Clin Obstet Gynecol 2017;60(1):206-214. https://doi.org/10.1097/GRF.0000000000000255

8. Witlin AG, Saade GR, Mattar F, Sibai BM. Risk factors for abruptio placentae and eclampsia: Analysis of 445 consecutively managed women with severe preeclampsia and eclampsia. Am J Obstet Gynecol
of 1999;180(6):1322-1329. https://doi.org/10.1016/S0002-9378(99)70014-

9. Sibai BM, Abdella TN, Anderson GD. Pregnancy outcomes in 211 patients with mild to moderate chronic hypertension. Obstet Gynecol 1983;61:571-576.

10. James PA, Oparil S, Carter BL, et al. Evidence based guideline for the management of high blood pressure in adults: Report from the panel members appointed to the Eighth Joint National Committee (JNC8). JAMA 2014;31(1):507-520. https://doi.org/10.1001/JAMA2013.284427

11. American College of Cardiology/American Heart Association Task Force on Clinical Practice Guidelines. Guideline for the prevention, detection, evaluation, and management of high blood pressure in adults. 2017. https://www.acc.org/ /media/Non-Clinical/Files-PDFs-Excel-MS-Word-etc/ Guidelines/2017/Guidelines_Made_Simple_2017_HBP.pdf (accessed 11 February 2018).

12. Bakris G, Sorrentino M. Redifining hypertension - assessment of the new blood pressure guidelines. N Engl J Med 2018;378(6):497-499. https://doi.org/10.1056/NEJMp1716193

13. Rowe H, Baker T, Hale TW. Maternal medication, drug use, and breastfeeding. Child Adolesc Psychiatr Clin N Am 2014;24(1):1-20. https://doi.org/10.1016/j.chc.2014.09.005

Accepted 4 May 2018. 